\title{
Autécologie du chêne tauzin (Quercus pyrenaica Willd.) en Galice (Espagne)
}

\author{
Ignacio Javier DíAZ-MARoto*, José FernándeZ-PARAJES, Pablo VILA-LAMEIRO \\ Departamento de Enxeñería Agroforestal, Universidade de Santiago de Compostela, Campus Universitario s/n, \\ 27002 Lugo, Espagne
}

(Reçu le 14 mars 2005; accepté le 8 juillet 2005)

\begin{abstract}
Résumé - Le présent travail traite de l'étude des « rebollares » (bois de Quercus pyrenaica Willd.) en Galice, tant d'un point de vue écologique que dendrométrique. Pour cela nous avons réalisé une caractérisation paramétrique des biotopes que cette espèce occupe actuellement. Nous avons installé des parcelles en 40 points de l'échantillonnage et nous avons élaboré 33 paramètres écologiques caractéristiques du biotope, ainsi que 14 paramètres dendrométriques et sylvicoles. Ceci nous a permis de déterminer l'habitat topographique, climatique et édaphique, à la fois central et marginal, de Quercus pyrenaica en Galice, ainsi que d'évaluer la situation actuelle des peuplements qu'ils occupent d'un point de vue sylvicole. Il s'agit de forêts plutôt xérophiles, moins productives que les chênaies de Quercus robur et de $Q$. petraea, ce qui explique qu'elles soient plus exposées aux incendies. Ceci provoque la dégradation de la plupart d'entre elles, accentuée par l'abandon des traditionnelles exploitations de bois de chauffage et de charbon végétal. C'est pourquoi il est difficile de trouver de bons peuplements de bois de chênes tauzins, ainsi que des pieds de taille élevée. Ils s'installent à une altitude de variation supérieure à $1000 \mathrm{~m}$, ce qui met en évidence une différence de température moyenne supérieure à $6^{\circ} \mathrm{C}$. Les précipitations annuelles moyennes sont de $1067,5 \mathrm{~mm}$, la moyenne estivale est de $112,7 \mathrm{~mm}$, ce qui signale une sécheresse estivale à certains endroits.
\end{abstract}

\section{Quercus pyrenaica / autécologie / biotope / limite écologique / Galice}

\begin{abstract}
Autecology of rebollo oak (Quercus pyrenaica Willd.) in Galicia (Spain). The present work was focused as an ecological and tree mensuration Quercus pyrenaica stands study in Galicia. With that objective, a parametric characterization of the species actual located biotypes was made measuring 40 plots and 33 ecological parameters and another 14 tree mensuration and sylvicultural ones. This data has allowed to determine physiographic, climatic and edaphic habitat, central and marginal, of Quercus pyrenaica in Galicia. The results shows that this forests are more dry and less productive than Quercus robur and Q. petraea stands, what means abandonment of the traditional uses like firewood and a great fire risk with its sequential degradation. This situation involves a lot difficult to find good stands of Quercus pyrenaica. As summary it could be said that this forests present an altitudinal variation higher to $1000 \mathrm{~m}$, what means an average temperature difference near to $6{ }^{\circ} \mathrm{C}$, and the annual precipitation average is $1067.5 \mathrm{~mm}$, being the summer average $115.2 \mathrm{~mm}$, with somewhere summer drought.
\end{abstract}

\section{Quercus pyrenaica / autecology / site / ecologic limit / Galicia}

\section{INTRODUCTION}

Le «rebollo » (Quercus pyrenaica Willd.) présente une distribution méditerranéenne-occidentale qui s'étend sur une grande partie du sud-ouest de la France, jusqu'en Bretagne, la péninsule ibérique et le nord-est du Maroc [4, 14, 43, 44]. La péninsule ibérique est le territoire qu'il occupe de manière prédominante entre les $9^{\circ} 20^{\prime}$ Ouest, en Extramadoure portugaise jusqu'à près des $3^{\circ}$ Est. En Galice, de grands peuplements sont présents dans la province d'Ourense et dans la moitié sud de Lugo (Fig. 1). On le trouve aussi sur le reste du territoire, le plus souvent mélangé au Quercus robur, avec lequel, il entre en contact le long d'une large bande de limite naturelle. Il forme de cette manière des forêts mixtes, et s'hybride facilement, donnant naissance au Quercus $\times$ andegavensis Hy [20,51].
Les chênaies de chêne tauzin présentent une gamme étendue de sous-types climatiques, avec une nette préférence pour les némoral-méditerranéens $[1,2]$. Les particularités biologiques de l'espèce en Galice nous permettent de lui attribuer un rôle de transition entre les chênes européens (Quercus robur et $Q$. petraea) et les espèces typiquement méditerranéennes du genre Quercus [43]. Dans le reste de la péninsule ibérique, c'est le chêne faginé (Quercus faginea) qui occupe ce caractère transitionnel, et le chêne pubescent (Quercus pubescens), dans le reste de l'Europe, en particulier en France [4, 14, 16]. À l'intérieur de la péninsule ibérique, dans la plus grande partie de la moitié ouest, les chênaies de Quercus pyrenaica préfèrent des zones où les précipitations annuelles sont supérieures à $725 \mathrm{~mm}$ et les précipitations estivales supérieures à $125 \mathrm{~mm}$. La température moyenne du mois de janvier est comprise entre -5 et $7{ }^{\circ} \mathrm{C}$,

* Auteur pour correspondance : diazmaro@lugo.usc.es 


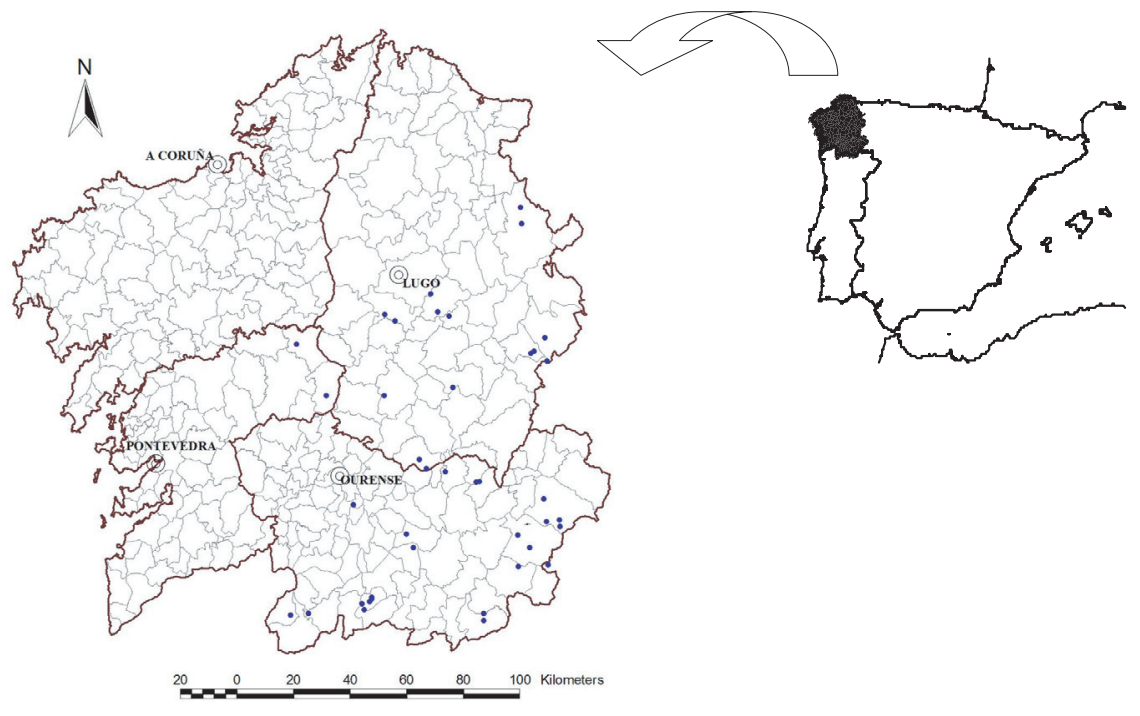

Figure 1. Situation du domaine d'étude (Communauté Autonome de Galice) et des parcelles d'échantillonnage dans le contexte de la Péninsule Ibérique.

celle du mois d'août, entre 12 et $25^{\circ} \mathrm{C}$ [16]. Il présente une grande résistance au climat continental et au froid, grâce à une feuillaison tardive et à un cycle court [2, 14, 29]. Les bois de chênes tauzins galiciens subsistent des précipitations annuelles qui oscillent entre 750 et $1400 \mathrm{~mm}$, les précipitations estivales étant comprises entre 60 et $170 \mathrm{~mm}$. La température moyenne du mois le plus froid, atteint les $-4{ }^{\circ} \mathrm{C}$, et celle du mois le plus chaud se rapproche des $26^{\circ} \mathrm{C}$.

Les roches mères sur lesquelles s'établissent ces forêts sont très variées. Dans leur majeure partie, elle sont siliceuses (granites, gneiss, schistes, quartz et ardoises), et dans certaines zones à précipitations élevées, elles se développent sur les calcaires décalcifiés et des dolomites [3, 14, 45]. La gamme des sols est également étendue, surtout les cambisols et les rankers $[3,59]$. En plusieurs points de la zone de distribution française, ces forêts s'établissent sur des néo-luvisols dystriques à humus de type moder, dysmoder et, y compris mor [35]. En Galice, elles présentent aussi une roche mère siliceuse, s'agissant du type de sol le plus représentatif [22] : régosols sombriques, brunisols humiques et cambisols dystriques.

Le chêne tauzin possède un tempérament robuste, pouvant se qualifier d'espèce de demi-ombre. Au cours de ses premières phases de développement, les semis nécessitent beaucoup de lumière et exigent plus d'abris au cours des saisons les plus extrêmes [14, 16, 29]. Au sein de la zone d'étude, il présente une croissance relativement lente [60] et il ne parvient pas à atteindre la taille du Quercus robur ou du $Q$. petraea, bien que sur les bonnes stations forestières il puisse dépasser les $20 \mathrm{~m}$ de hauteur, et atteindre presque les $30 \mathrm{~m}$ [29]. Son système racinaire est formé de deux types de racines : une racine principale pivotante qui lui permet un puissant ancrage au terrain et de nombreuses racines secondaires proches de la surface et parallèles à celle-ci, capables de donner naissance à des drageons en grande profusion formant un dense fourré dans les taillis [3, 8 , $14,16,58]$. La dégradation subie par ces forêts, provoquée par les incendies forestiers, les traitements sylvicoles comme la taille en têtard des arbres, ou l'abandon de l'exploitation traditionnelle du bois de chauffage et du charbon végétal, ou encore, l'évolution naturelle à la suite d'un incendie, nous mène, dans la plupart des cas, à de jeunes peuplements abandonnés, de densité élevée, et qui présentent une perte de vigueur due à la forte concurrence existante. Ceci se traduit le plus fréquemment par une sécheresse des extrémités des drageons [3, 8, 29].

En tout, onze associations phytosociologiques ont été décrites parmi lesquelles se trouve Quercus pyrenaica [51, 65], ainsi qu'une association française et une autre marocaine [43]. Quant à la surface qu'elles occupent au sein de la péninsule ibérique, seules six d'entre elles se caractérisent comme une entité suffisante : Linario triornithophorae-Quercetum pyrenaicae RivasMartínez et al., 1984, Holco molli-Quercetum pyrenaicae Br.B1., P. Silva \& Rozeira 1956, Genisto falcatae-Quercetum pyrenaicae Rivas-Martínez in Penas \& Díaz 1985, lesquelles sont aussi présentes en Galice [59], Luzulo forsteri-Quercetum pyrenaicae Rivas-Martínez 1963, Arbuto unedis-Quercetum pyrenaicae Rivas-Martínez 1987 et Festuco heterophyllaeQuercetum pyrenaicae Br.-B1. 1967 [51].

Les peuplements de Quercus pyrenaica occupent en Galice 100504 ha, soit plus de $7 \%$ de la surface boisée [19], on peut remarquer sa progression puisqu'en 1986 ils n'occupaient que 31459 ha [68]. Cette surface est légèrement inférieure à celle qui existait en 1972, de 35181 ha [37]. En général, en Galice, cette augmentation est principalement due à la réduction de la pression anthropique qui s'exerce sur les chênes, ainsi qu'à l'abandon des exploitations forestières et à une diminution de incendies [19, 54]. D'ailleurs, ces bois sont des habitats d'intérêt communautaire (Directive 92/43/CEE), de là l'importance de leur conservation, pour laquelle, il est nécessaire de se situer au niveau régional et d'avancer dans la connaissance des exigences écologiques du Quercus pyrenaica. Les objectives de ce travail sont : (1) la caractérisation des écosystèmes de l'espèce en Galice, à partir de divers paramètres écologiques ; (2) la détermination de son habitat topographique, climatique et édaphique ; (3) rapporter les paramètres écologiques avec les dendrométriques et sylvicoles, indicateurs de l'usage et de l'état de conservation de ces forêts. 
Tableau I. Caractéristiques topographiques des 40 parcelles échantillonnées.

\begin{tabular}{|c|c|c|c|c|c|c|}
\hline $\begin{array}{l}\text { Nombre } \\
\text { parcelle }\end{array}$ & $\begin{array}{c}\text { Nom } \\
\text { parcelle }\end{array}$ & $\begin{array}{c}\text { Code } \\
\text { parcelle }\end{array}$ & Province & $\begin{array}{l}\text { Altitude } \\
\text { (m) }\end{array}$ & $\begin{array}{l}\text { Pente } \\
(\%)\end{array}$ & Orientation \\
\hline 1 & A Golada & OR & Pontevedra & 532 & 34 & Sud-ouest \\
\hline 2 & A Gudiña & $\mathrm{AG}$ & Ourense & 983 & 7 & Nord-est \\
\hline 3 & A Veiga I & MD & Ourense & 956 & 27 & Sud-ouest \\
\hline 4 & A Veiga II & $\mathrm{SN}$ & Ourense & 1095 & 29 & Nord-est \\
\hline 5 & As Nogais & NO & Lugo & 886 & 60 & Nord \\
\hline 6 & Castro Caldelas & $\mathrm{CC}$ & Ourense & 550 & 39 & Sud \\
\hline 7 & Corgo & TO & Lugo & 387 & 0 & - \\
\hline 8 & Covas & $\mathrm{CO}$ & Ourense & 1054 & 14 & Sud-ouest \\
\hline 9 & Doiras & DO & Lugo & 627 & 49 & Nord-ouest \\
\hline 10 & Entrimo & ET & Ourense & 559 & 6 & Sud-est \\
\hline 11 & Escairón & $\mathrm{CZ}$ & Lugo & 450 & 29 & Nord-ouest \\
\hline 12 & A Fonsagrada I & FO & Lugo & 602 & 55 & Sud \\
\hline 13 & A Fonsagrada II & TR & Lugo & 660 & 63 & Nord-ouest \\
\hline 14 & Guntín I & $\mathrm{FE}$ & Lugo & 485 & 30 & Sud-ouest \\
\hline 15 & Guntín II & VM & Lugo & 414 & 34 & Sud \\
\hline 16 & Láncara & $\mathrm{NE}$ & Lugo & 454 & 14 & Nord-ouest \\
\hline 17 & Muiños & MU & Ourense & 621 & 42 & Est \\
\hline 18 & O Curro & $\mathrm{CU}$ & Lugo & 1172 & 0 & - \\
\hline 19 & O Incio & OI & Lugo & 576 & 17 & Sud-ouest \\
\hline 20 & Os Blancos I & $\mathrm{CA}$ & Ourense & 1136 & 20 & Sud-ouest \\
\hline 21 & Os Blancos II & OB & Ourense & 824 & 22 & Sud-est \\
\hline 22 & Os Blancos III & TB & Ourense & 862 & 31 & Sud-ouest \\
\hline 23 & Pedradita & $\mathrm{PE}$ & Lugo & 1273 & 7 & Nord-ouest \\
\hline 24 & Pexeiros & PX & Ourense & 1005 & 26 & Sud-est \\
\hline 25 & Pías & PI & Ourense/Zamora & 1205 & 16 & Nord-ouest \\
\hline 26 & Ramilo & RA & Ourense & 1125 & 25 & Nord-ouest \\
\hline 27 & Rios & RI & Ourense & 856 & 20 & Sud \\
\hline 28 & Rodeiro & $\mathrm{RO}$ & Pontevedra & 772 & 0 & - \\
\hline 29 & San Cibrao das Viñas & $\mathrm{CV}$ & Ourense & 300 & 0 & - \\
\hline 30 & San Xoan do Rio I & SX & Ourense & 813 & 45 & Ouest \\
\hline 31 & San Xoan do Rio II & PR & Ourense & 720 & 40 & Nord \\
\hline 32 & Sober I & $\mathrm{AM}$ & Lugo & 474 & 0 & - \\
\hline 33 & Sober II & $\mathrm{BL}$ & Lugo & 516 & 13 & Sud \\
\hline 34 & Santa Comba & $\mathrm{SC}$ & Lugo & 429 & 11 & Ouest \\
\hline 35 & Valdín & $\mathrm{RC}$ & Ourense & 1311 & 0 & - \\
\hline 36 & Viana do Bolo I & VB & Ourense & 990 & 41 & Nord-ouest \\
\hline 37 & Viana do Bolo II & $\mathrm{BO}$ & Ourense & 715 & 46 & Ouest \\
\hline 38 & Vilar de Barrio I & $\mathrm{AR}$ & Ourense & 598 & 20 & Sud \\
\hline 39 & Vilar de Barrio II & VI & Ourense & 927 & 33 & Sud-ouest \\
\hline 40 & Vilardevós & VV & Ourense & 1032 & 20 & Nord-ouest \\
\hline
\end{tabular}

\section{MATÉRIELS ET MÉTHODES D'ÉTUDE}

\subsection{Positionnement de l'étude et échantillonnage}

La zone d'étude est située dans le nord-ouest de la péninsule Ibérique et comprend la Communauté Autonome de la Galice (Espagne) (Fig. 1).

Du fait de la dispersion et de la variété des chênaies à l'intérieur du domaine d'étude, nous n'avons pas réalisé de stratification initiale du territoire [9, 20,21,27], c'est pourquoi, la zone étudiée a été considéré dans sa totalité [18]. En nous basant sur la carte forestière de l'Espagne [54] nous avons sélectionné les points d'échantillonnage à l'aide de l'information que nous a facilité le personnel de l'administration forestière, ainsi que de celle recueillie au cours de travaux précédents $[19,29]$. Au début, nous avons projeté de considérer une surface minimum comprise entre 0,5 et 1 ha, permettant d'installer des parcelles d'inventaire en évitant les problèmes liés à l'effet de bordure $[20,29,36]$.

Une fois les peuplements repérés, nous avons installé un dispositif de parcelles d'échantillonnage rectangulaires, de dimensions variables, en fonction du nombre de pieds. De cette façon, les arbres atteignant un diamètre supérieur au minimum requis pour l'inventaire (diamètre normal égal ou supérieur à $5 \mathrm{~cm}$ ) ne se situent pas au-dessous de $50[36,52]$, ce qui représente une surface de parcelle comprise entre 130 et $2120 \mathrm{~m}^{2}$. Le nombre total de parcelles sélectionnées a été de 40 (Tab. I). Une fois implantées, nous avons réalisé l'inventaire en relevant les données topographiques, dendrométriques et les caractéristiques du sol. Munis de ces informations, ainsi que les données climatologiques adaptées aux différents points de l'échantillonnage, suivant la méthodologie de Carballeira et al. [12], et des résultats des analyses édaphiques, nous avons élaboré une série de paramètres sur 
chaque parcelle répondant aux caractéristiques de l'habitat (topographique, climatique et édaphique) de Quercus pyrenaica en Galice, et de l'état sylvicole de ces bois [7, 21, 29].

\subsection{Paramètres pris en compte}

Un total de 47 paramètres a été relevé (Tab. II). Trente-trois sont écologiques - topographiques (6), climatiques (10) et édaphiques (17) - $[15,40,53]$, les 14 autres sont des paramètres qui caractérisent les bois de Quercus pyrenaica tant à niveau dendrométrique que sylvicole $[7,21,29,41]$.

Concernant les paramètres édaphiques, nous avons calculé leur valeur « superficielle » et totale selon le profil édaphique, excepté le pourcentage de gravier total. Lorsque nous avons relevé la valeur totale de chaque paramètre en suivant l'ensemble du profil édaphique, nous avons considéré la moyenne pondérée de tout le profil, en appliquant la méthode de Russell et Moore [55]. Concernant les valeurs superficielles, nous avons relevé les données sur une profondeur de $20 \mathrm{~cm}$ à partir de la surface du sol, excepté pour les cas où il existait plus d'un horizon édaphique sur cette épaisseur. Il a donc été nécessaire alors, de calculer une moyenne pondérée $[21,27,53]$.

\subsection{Analyse statistique}

Avec l'ensemble des paramètres calculés, nous avons élaboré une base de données nous informant sur le biotope et les particularités dendrométriques et sylvicoles des forêts de Quercus pyrenaica en Galice. Nous avons analysé celle-ci de forme univariable [66], ce qui nous a permis de calculer une série de valeurs caractéristiques [21,27, 53]: Limite Inférieure (LI), Seuil Inférieur (SI) : percentile 10, Moyenne (M), Seuil Supérieur (SS) : percentile 90 et Limite Supérieure (LS), grâce auxquels il est possible de délimiter le spectre écologique et de définir l'habitat du Quercus pyrenaica, en accord avec les critères suivants [21, 23, 27] :

1. Qualifier comme habitat central ou optimum, par rapport à un paramètre, l'intervalle défini par les Seuils Supérieur et Inférieur, formé par $80 \%$ des parcelles étudiées.

2. Considérer comme habitat marginal, par rapport à un paramètre, les intervalles compris entre la Limite et le Seuil Inférieur et entre le Seuil et la Limite Supérieure, formé par $20 \%$ des parcelles.

Ils sont tous deux considérés comme une première approche pour décrire la phytocoénose des forêts de chênes tauzins en Galice, étant donné que tous les paramètres, n'auront pas la même signification en tant que descripteurs de l'habitat de ces formations [9, 21, 23, 27]. Pour cela, il a été nécessaire de réaliser une analyse discriminante des parcelles afin d'identifier les paramètres possédant un plus grand poids descriptif $[34,39,42,61]$. Nous avons utilisé la méthodologie proposée par Hill et al. [33], qui nous a permis d'obtenir une classification dichotomique en fonction de certains caractéristiques des parcelles d'échantillonnage prises au hasard : la présence d'espèces végétales $[27,33]$, des valeurs de paramètres écologiques et sylvicoles [5, 20], en employant le programme Twinspan [31, 32, 49].

Par la suite, nous avons réalisé une analyse bivariable, [57] entre l'information sylvicole et les paramètres, à la fois dendrométriques/ sylvicoles et écologiques [17, 29]. Ceci afin de détecter dans un premier temps, la possible inter-relation entre les paramètres, et afin d'évaluer, dans un deuxième temps, l'influence du facteur écologique sur les paramètres sylvicoles, à travers de l'analyse de variance à sens unique selon le Multiple Range Test [57].

\section{RÉSULTATS}

L'information concernant le biotope et les caractéristiques dendrométriques et sylvicoles des bois de Quercus pyrenaica apportée par les paramètres pris en compte, nous sert de base pour décrire l'habitat du chêne tauzin, à une échelle régionale, la Galice. Pour cela, les figures 2, 3 et 4 relèvent la description statistique à variable unique que nous fournissent les paramètres
Tableau II. Paramètres pris en compte.

\begin{tabular}{|c|c|c|}
\hline Numéro & Paramètre (unités) & $\begin{array}{c}\text { Code } \\
\text { paramètre }\end{array}$ \\
\hline 1 & Altitude moyenne (m) & ALT \\
\hline 2 & Pente moyenne $(\%)$ & PND \\
\hline 3 & Orientation $\left({ }^{\circ}\right)$ & ORI \\
\hline 4 & ${ }^{\mathrm{a}}$ Insolation & INS \\
\hline 5 & Profondeur du sol jusqu'à la roche mère $(\mathrm{cm})$ & PRO \\
\hline 6 & Distance à la mer en suivant une ligne droite $(\mathrm{km})$ & DM \\
\hline 7 & Précipitations annuelles totales (mm) & PT \\
\hline 8 & Précipitations estivales (mm) & PE \\
\hline 9 & Température annuelle moyenne $\left({ }^{\circ} \mathrm{C}\right)$ & $\mathrm{TM}$ \\
\hline 10 & Température annuelle moyenne des maxima absolus $\left({ }^{\circ} \mathrm{C}\right)$ & TMA \\
\hline 11 & Température annuelle moyenne des minima absolus $\left({ }^{\circ} \mathrm{C}\right)$ & $\mathrm{TmA}$ \\
\hline 12 & Évapotranspiration annuelle selon ${ }^{\mathrm{b}}$ Thornthwaite (mm) & ETP \\
\hline 13 & c Somme des excédents (mm) & SUP \\
\hline 14 & ${ }^{\mathrm{c}}$ Somme des déficits $(\mathrm{mm})$ & DEF \\
\hline 15 & ${ }^{\mathrm{d}}$ Indice hydrique & $\mathrm{IH}$ \\
\hline 16 & e Durée de la sécheresse (nombre de mois) & DSQ \\
\hline 17 & $\mathrm{pH}$ total $\mathrm{H}_{2} \mathrm{O}$ & PH \\
\hline 18 & $\mathrm{pH}$ superficiel $\mathrm{H}_{2} \mathrm{O}$ & PHS \\
\hline 19 & Matière organique totale $(\%)$ & MO \\
\hline 20 & Matière organique superficielle $(\%)$ & MOS \\
\hline 21 & Azote total $(\%)$ & $\mathrm{N}$ \\
\hline 22 & Azote superficiel $(\%)$ & NS \\
\hline 23 & Rapport carbone-azote total & $\mathrm{C} / \mathrm{N}$ \\
\hline 24 & Rapport carbone-azote superficiel & $\mathrm{C} / \mathrm{NS}$ \\
\hline 25 & Phosphore assimilable total (ppm) & $\mathrm{P}$ \\
\hline 26 & Phosphore assimilable superficiel (ppm) & PS \\
\hline 27 & Potassium changeable total (ppm) & $\mathrm{K}$ \\
\hline 28 & Potassium changeable superficiel (ppm) & KS \\
\hline 29 & Calcium changeable total (ppm) & $\mathrm{Ca}$ \\
\hline 30 & Calcium changeable superficiel (ppm) & $\mathrm{CaS}$ \\
\hline 31 & Magnésium changeable total (ppm) & $\mathrm{Mg}$ \\
\hline 32 & Magnésium changeable superficiel (ppm) & $\mathrm{MgS}$ \\
\hline 33 & Gravier total (éléments > 2 mm) $(\%)$ & GRA \\
\hline 34 & Nombre de pieds par hectare $\left(\mathrm{No} \mathrm{ha}^{-1}\right)$ & DEN \\
\hline 35 & Surface terrière par hectare $\left(\mathrm{m}^{2} \mathrm{ha}^{-1}\right)$ & $\mathrm{ABA}$ \\
\hline 36 & Diamètre moyen arithmétique $(\mathrm{cm})$ & DMA \\
\hline 37 & Diamètre moyen quadratique $(\mathrm{cm})$ & DMC \\
\hline 38 & Déviation standard de la distribution diamétrale $(\mathrm{cm})$ & DED \\
\hline 39 & Coefficient de variation de la distribution diamétrale (\%) & CVD \\
\hline 40 & Diamètre dominant $(\mathrm{cm})$ & DOM \\
\hline 41 & Hauteur moyenne arithmétique (m) & HMA \\
\hline 42 & Hauteur moyenne quadratique $(\mathrm{m})$ & HMC \\
\hline 43 & Déviation standard de la distribution des hauteurs (m) & DEA \\
\hline 44 & Coefficient de variation de la distribution des hauteurs (\%) & CVA \\
\hline 45 & ${ }^{\mathrm{f}}$ Hauteur dominante $(\mathrm{m})$ & HDA \\
\hline 46 & Indice de Hart (\%) & IHA \\
\hline 47 & ${ }^{\mathrm{g}}$ Indice de Czarnowski & ICZ \\
\hline
\end{tabular}

a Insolation [26]; b Thornthwaite [64]; c Bilan hydrique (somme des excédents et des déficits, différences positives et négatives, entre les précipitations et l'ETP mensuel); d Indice hydrique selon Thornthwaite et

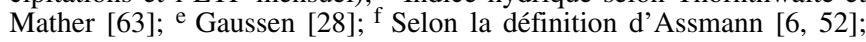
g Nombre de pieds que contient une parcelle carrée dont le côté correspond à la HMA. 


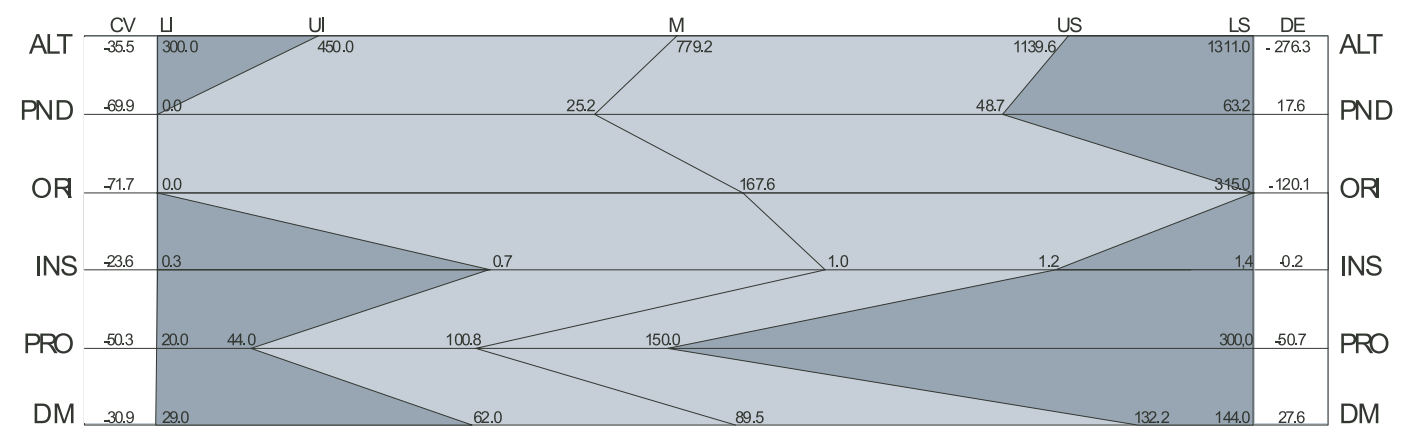

Figure 2. Statistiques descriptives et habitat topographique central et marginal du Quercus pyrenaica en Galice.

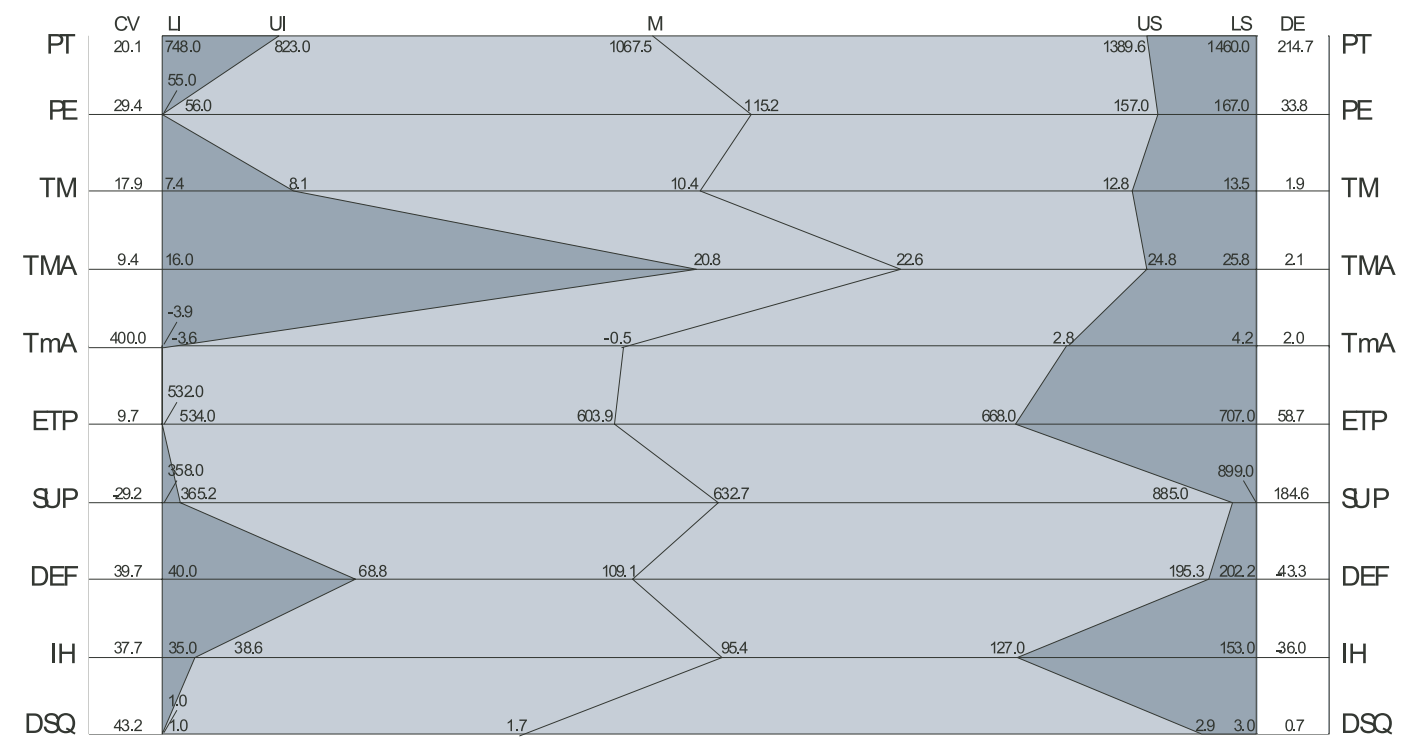

Figure 3. Statistiques descriptives et habitat climatique central et marginal du Quercus pyrenaica en Galice.

écologiques et les habitats topographique, climatique et édaphique, aussi bien centraux que marginaux. Afin d'évaluer, d'un point de vue sylvicole, la situation actuelle des chênes tauzins, le tableau III reflète les statistiques descriptives des paramètres dendrométriques et sylvicoles.

L'analyse discriminante des parcelles a donné lieu à onze groupes (Tab. IV). Les paramètres discriminants obtenus, lors de la caractérisation de ces groupes, correspondent aux paramètres de nature topographique (ALT, PRO et DM), climatique (PE, TMA) et, y compris ceux de nature édaphique (P).

$\mathrm{Au}$ cours d'une première analyse bivariable, les résultats obtenus nous ont permis de sélectionner les paramètres dendrométriques/sylvicoles (Tab. V), dont la relation était la plus significative, comme suit : DED, DEA, IHA, DOM, DEN, DMA, DMC et HDA. La seconde analyse bivariable (Tab. VI), nous a permis de confronter les paramètres sylvicoles et les paramètres discriminants obtenus durant le suivi de la méthode Twinspan (ALT, PRO, DM, PE, TMA et P). Le résultat met en évidence une étroite relation avec le paramètre d'altitude et, à moindre mesure, avec les paramètres de distance à la mer et de précipitations estivales.

En dernier lieu, les deux paramètres discriminants présentant une plus grande corrélation (ALT et PE), ont été confrontés aux paramètres sylvicoles sélectionnés (DEN, DMA, DMC et DOM), à travers d'une analyse de variance. Les résultats les plus significatifs ont été (Fig. 5) : (1) Une densité plus importante du nombre de pieds et, en conséquence, une diminution des valeurs diamétrales en augmentant l'altitude ; (2) une réduction du diamètre des arbres pour des valeurs de précipitations estivales inférieures à 125 et supérieures à $150 \mathrm{~mm}$.

\section{DISCUSSION}

Concernant les paramètres topographiques et climatiques, à coefficient de variation supérieurs à $50 \%$, la variabilité la plus nette apparaît auprès des paramètres PND, ORI, PRO et Tma (Fig. 2). Si nous analysons séparément ces données, en commençant par la pente, la valeur obtenue nous indique que les bois de chênes 


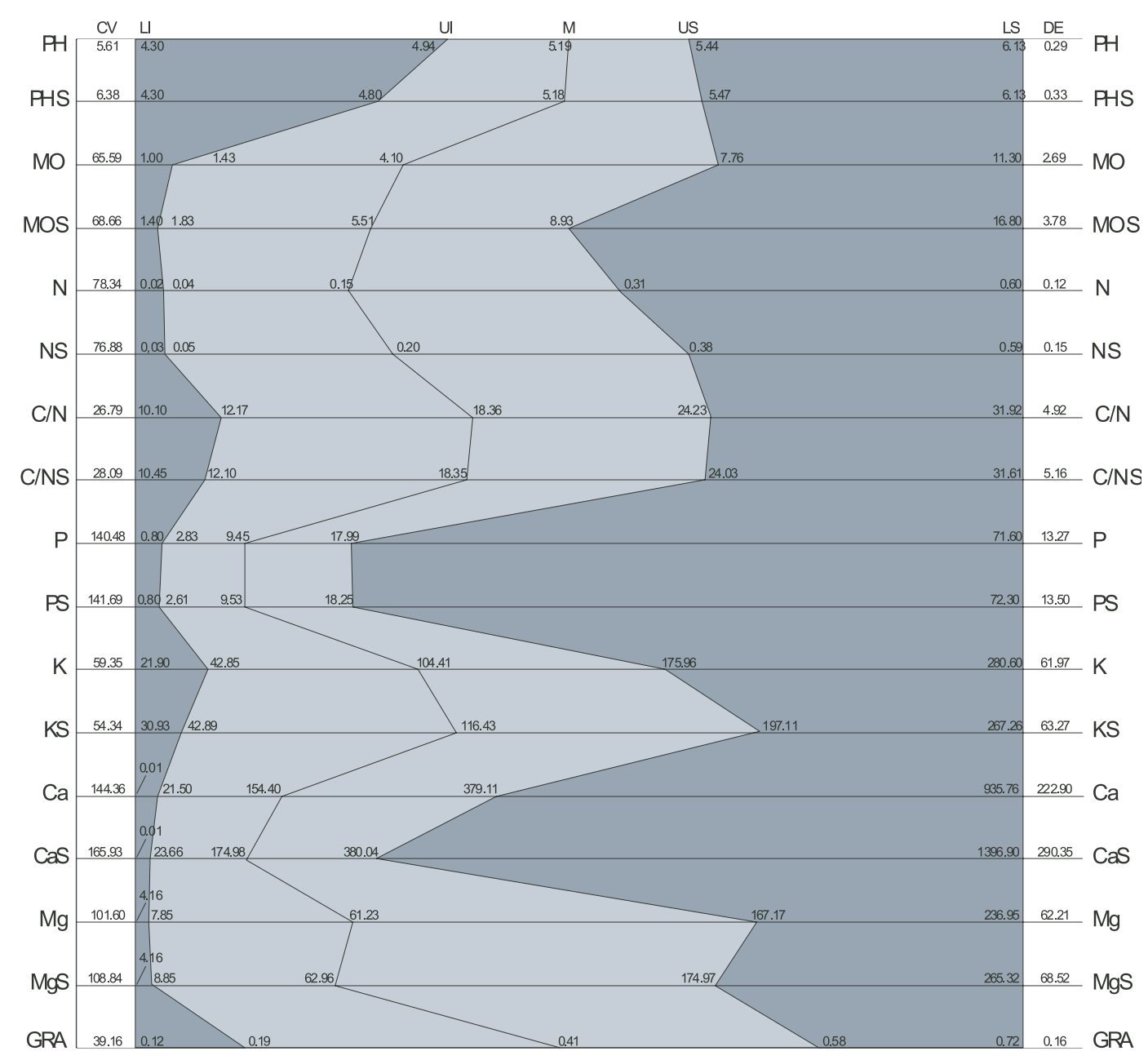

Figure 4. Statistiques descriptives et habitat édaphique central et marginal du Quercus pyrenaica en Galice.

Tableau III. Statistiques descriptives des paramètres dendrométriques/sylvicoles ( $n=40)$ (DE : déviation standard, CV : coefficient de variation).

\begin{tabular}{|c|c|c|c|c|c|}
\hline Paramètre & Moyenne & $\mathrm{DE}$ & $\mathrm{CV}(\%)$ & Maximale & Minimale \\
\hline DEN $\left(\mathrm{No} \mathrm{ha}^{-1}\right)$ & 1711,5 & 1155,0 & 67,5 & 4230,8 & 259,4 \\
\hline $\operatorname{ABA}\left(\mathrm{m}^{2} \mathrm{ha}^{-1}\right)$ & 27,6 & 7,5 & 27,3 & 42,3 & 11,9 \\
\hline DMA $(\mathrm{cm})$ & 16,4 & 6,7 & 41,0 & 29,9 & 8,2 \\
\hline $\mathrm{DMC}(\mathrm{cm})$ & 17,5 & 7,1 & 40,6 & 31,4 & 8,5 \\
\hline $\mathrm{DED}(\mathrm{cm})$ & 6,0 & 2,8 & 46,3 & 12,9 & 1,7 \\
\hline CVD (\%) & 0,4 & 0,1 & 25,7 & 0,6 & 0,2 \\
\hline DOM (cm) & 26,8 & 9,0 & 33,6 & 42,1 & 12,3 \\
\hline HMA (m) & 11,8 & 3,6 & 30,3 & 20,6 & 6,1 \\
\hline HMC (m) & 12,4 & 3,7 & 30,0 & 21,5 & 6,3 \\
\hline DEA (m) & 3,6 & 1,3 & 35,7 & 6,1 & 0,7 \\
\hline CVA $(\%)$ & 0,3 & 0,1 & 24,4 & 0,4 & 0,1 \\
\hline HDA (m) & 15,7 & 3,8 & 24,4 & 24,5 & 7,6 \\
\hline $\operatorname{IHA}(\%)$ & 7,2 & 6,5 & 89,5 & 41,5 & 2,6 \\
\hline ICZ & 18,4 & 7,8 & 42,1 & 33,4 & 5,8 \\
\hline
\end{tabular}

Voir liste des paramètres dans tableau II. 
Tableau IV. Groupes finaux résultants de l'application du programme Twinspan.

\begin{tabular}{lcc}
\hline Groupe & Paramètres discriminants & Parcelles \\
\hline A & TMA $>$ M+DE & $1,11,32,33$ \\
B & M-DE $<$ TMA $<$ M+DE & $10,17,38,39$ \\
C & $\mathrm{DM}>$ M+DE & 26,36 \\
D & M-DE $<$ DM $<$ M+DE & $14,15,30$ \\
E & $\mathrm{PE}>\mathrm{M}+\mathrm{DE}$ & $5,9,12,13,18,23$ \\
F & $\mathrm{M}-\mathrm{DE}<\mathrm{PE}<\mathrm{M}+\mathrm{DE}$ & 27,40 \\
$\mathrm{G}$ & $\mathrm{PE}<\mathrm{M}-\mathrm{DE}$ & $8,20,21,22,24,29$ \\
$\mathrm{H}$ & $\mathrm{P}>\mathrm{M}+\mathrm{DE}$ & 3,37 \\
& $\mathrm{M}-\mathrm{DE}<\mathrm{P}<\mathrm{M}+\mathrm{DE}$ & \\
$\mathrm{I}$ & $\mathrm{M}-\mathrm{DE}<\mathrm{ALT}<\mathrm{M}+\mathrm{DE}$ & $2,4,25,31,35$ \\
& $\mathrm{ALT}>\mathrm{M}+\mathrm{DE}$ & \\
$\mathrm{J}$ & $\mathrm{M}-\mathrm{DE}<\mathrm{P}<\mathrm{M}+\mathrm{DE}$ & $6,19,28,34$ \\
$\mathrm{~K}$ & $\mathrm{M}-\mathrm{DE}<\mathrm{ALT}<\mathrm{M}+\mathrm{DE}$ & 7,16 \\
& $\mathrm{PRO}<\mathrm{M}-\mathrm{DE}$ & \\
\hline
\end{tabular}

tauzins galiciens sont actuellement situés, pour beaucoup d'entre eux, dans des zones à versants prononcés, où ils ont demeuré grâce à une exploitation difficile liée à la topographie du terrain [8, 21]. Quant à l'orientation, le tableau I nous permet d'observer la grande variabilité qui existe au sein des parcelles inventoriées, avec une certaine préférence pour les orientations sud, bien que peu marquée, dû au tempérament robuste de l'espèce $[4,44]$. Les sols où s'établissent les bois de chênes tauzins sont généralement profonds, bien que les parcelles ayant souffert des incendies répétés, présentent des profondeurs de moindre importance $[8,29]$. Grâce à son système racinaire, ceci ne représente aucun problème pour le développement de l'espèce $[14,16]$. La température annuelle moyenne des minima absolus, est le seul paramètre climatique qui présente un coefficient de variation élevé, supérieur à $50 \%$. Ce comportement, conséquence d'une amplitude thermique élevée, accentuée par la variation d'altitude existante dans le domaine d'étude [12], a déjà été observé au cours d'un travail précédent, traitant de l'autécologie du Quercus robur en Galice [21]. À l'extrémité opposée, les paramètres PT, TM, TMA et ETP présentent un coefficient de variation inférieur à $20 \%$, ce qui met en évidence l'homogénéité des paramètres climatiques, à l'exception de la TmA, qui généralement est la preuve de l'existence d'un climat océanique humide, possédant une influence méditerranéenne à certains endroits $[2,47]$. La variabilité élevée de nombreux paramètres édaphiques [10, 46] (Fig. 4), est probablement due à l'éventail de roche-mères sur lesquelles s'établissent ces forêts, majoritairement siliceuses, donnant lieu à des sols acides dont le pH varie très peu (Fig. 4) [14, 45].

\subsection{Habitat topographique du Quercus pyrenaica en Galice}

En ce qui concerne l'habitat topographique central, il faut savoir que beaucoup de bois de chênes tauzins galiciens occupent des zones de coteaux moyens et élevés. Mais ils apparaissent tout aussi bien au fond des vallées, résultat de la pression anthropique (incendies de forêts, exploitation de forêt de chauffage et de charbon végétal, ainsi que de l'alimentation du bétail $[8,29])$. Ils s'installent en des lieux de moyenne et haute altitude à orientation variable, mais avec une préférence pour les zones à orientation sud, surtout dans la zone nord de l'étude. Cependant, vers le sud ils préfèrent l'ombre, conséquence

Tableau V. Coefficients de corrélation linéaire de Pearson entre les paramètres dendrométriques/sylvicoles.

\begin{tabular}{|c|c|c|c|c|c|c|c|c|c|c|c|c|c|c|}
\hline & DEN & ABA & DMA & DMC & DED & CVD & DOM & HMA & HMC & DEA & CVA & HDA & IHA & $\mathrm{ICZ}$ \\
\hline DEN & 1,000 & n.s. & $-0,854 * *$ & $-0,866^{* *}$ & $-0,782 * *$ & n.s. & $-0,831 * *$ & $-0,775^{* *}$ & $-0,784 * *$ & $-0,663 * *$ & n.s. & $-0,745^{* *}$ & $0,480 * *$ & $0,628 * *$ \\
\hline $\mathrm{ABA}$ & & 1,000 & $\mathrm{n} . \mathrm{s}$ & n.s. & n.s. & n.s. & n.s. & n.s. & n.s. & $0,364^{*}$ & $\mathrm{n} . \mathrm{s}$ & $0,322 *$ & $-0,372^{*}$ & $0,617 * *$ \\
\hline DMA & & & 1,000 & $0,996^{* *}$ & $0,797 * *$ & n.s. & $0,907 * *$ & $0,920 * *$ & $0,923^{* *}$ & $0,734 * *$ & n.s. & $0,843^{* *}$ & $-0,526^{* *}$ & $-0,432 * *$ \\
\hline DMC & & & & 1,000 & $0,846^{* *}$ & n.s. & $0,935^{* *}$ & $0,911 * *$ & $0,919 * *$ & $0,769 * *$ & n.s. & $0,853 * *$ & $-0,549 * *$ & $-0,450 * *$ \\
\hline DED & & & & & 1,000 & $0,561 * *$ & $0,936 * *$ & $0,692 * *$ & $0,726^{* *}$ & $0,861 * *$ & $0,428 * *$ & $0,766^{* *}$ & $-0,607 * *$ & $-0,477 * *$ \\
\hline CVD & & & & & & 1,000 & $0,361 * *$ & n.s. & n.s & $0,447 * *$ & $0,736^{* *}$ & n.s. & $-0,421 * *$ & n.s. \\
\hline DOM & & & & & & & 1,000 & $0,839 * *$ & $0,860 * *$ & $0,840 * *$ & n.s & $0,861 * *$ & $-0,632 * *$ & $-0,387 *$ \\
\hline HMA & & & & & & & & 1,000 & $0,998 * *$ & $0,736^{* *}$ & n.s. & $0,939 * *$ & $-0,562 * *$ & n.s \\
\hline HMC & & & & & & & & & 1,000 & $0,779 * *$ & n.s. & $0,953 * *$ & $-0,590 * *$ & n.s. \\
\hline DEA & & & & & & & & & & 1,000 & $0,614^{* *}$ & $0,858^{* *}$ & $-0,742 * *$ & n.s. \\
\hline CVA & & & & & & & & & & & 1,000 & n.s. & $-0,590 * *$ & n.s. \\
\hline HAD & & & & & & & & & & & & 1,000 & $-0,691 * *$ & n.s. \\
\hline IHA & & & & & & & & & & & & & 1,000 & n.s. \\
\hline $\mathrm{ICZ}$ & & & & & & & & & & & & & & 1,000 \\
\hline
\end{tabular}

Niveaux de signification (s) : $* \mathrm{~s}>95 \% ; * * \mathrm{~s}>99 \%$; n.s., non significatif. 

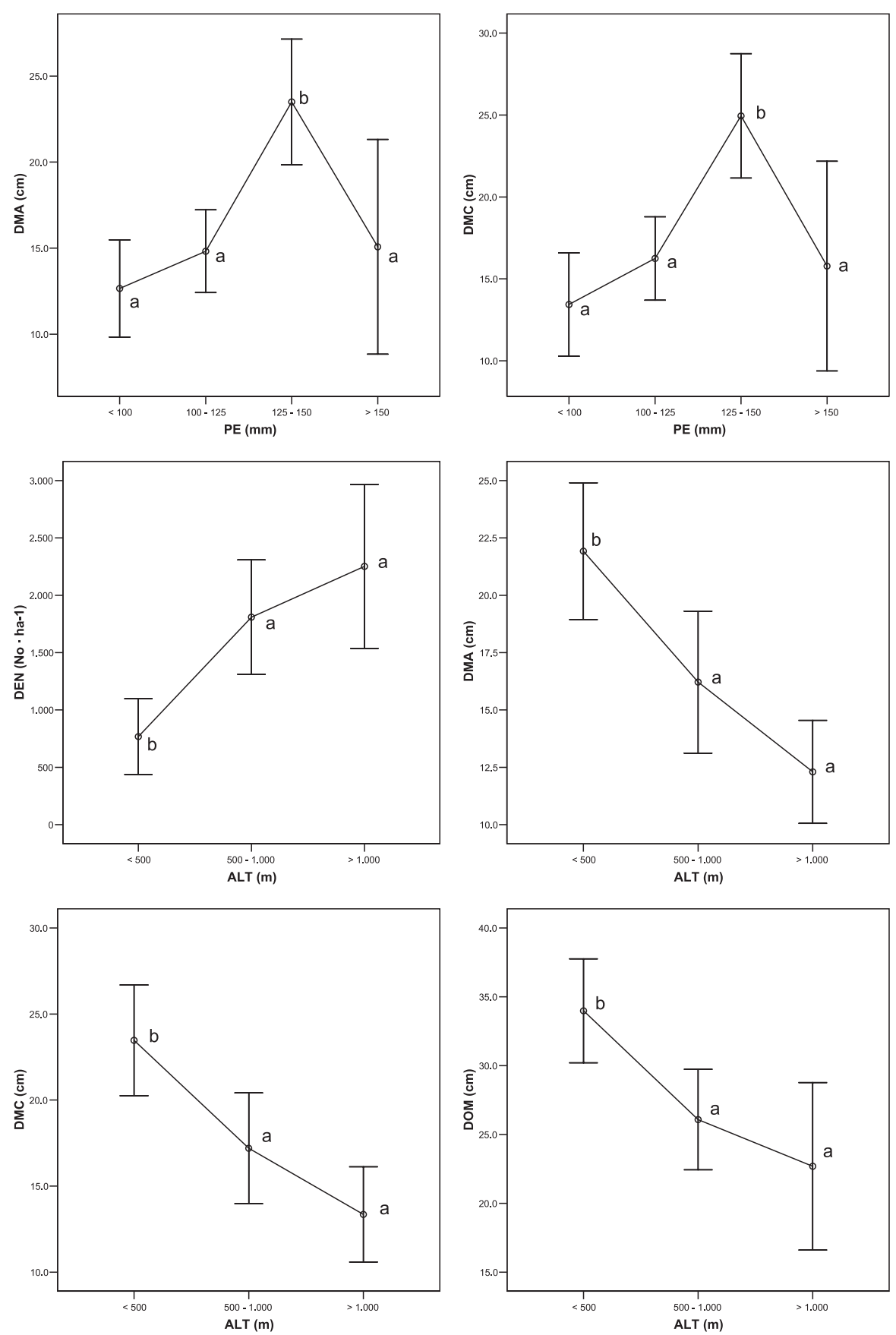

Figure 5. Analyse de variance entre l'altitude et les précipitations estivales avec les paramètres sylvicoles sélectionnés (les valeurs avec différents caractères sont statistiquement significatifs).

d'une plus grande influence méditerranéenne [16, 60] (Tab. I), ce qui correspond aux valeurs obtenues à travers le paramètre d'insolation (INS) [26] (Fig. 2). Bien que beaucoup de ces bois s'établissent sur des sols profonds (zones de talweg), d'autres s'établissent sur des sols de faible profondeur dans les zones de versant, où les incendies répétés ont occasionné une érosion du terrain. Quoi qu'il en soit, le Quercus pyrenaica s'adapte à l'une ou l'autre situation grâce aux deux types de racines qu'il possède $[3,14,16]$. Le paramètre de distance à la mer est com- pris entre 62 et $132 \mathrm{~km}$, en ligne droite (Fig. 2), ce qui coïncide avec la distribution potentielle de végétation en Galice [51, 60] et met en évidence le caractère plutôt méditerranéen de l'espèce $[2,43]$.

En ce qui concerne l'habitat topographique marginal, il est important de signaler l'existence de peuplements dans des zones relativement éloignées de leur domaine potentiel [51, 60]. Dans ces cas, il en découle, l'apparition des hybrides avec d'autres Quercus, comme le Quercus petraea dans les parcelles 
Tableau VI. Coefficients de corrélation linéaire de Pearson entre les paramètres dendrométriques/sylvicoles et les paramètres discriminants significatifs obtenus avec le programme Twinspan. (Les paramètres PRO, TMA et $\mathrm{P}$ ne sont pas significatifs.)

\begin{tabular}{lccc}
\hline & ALT & DM & PE \\
\hline DEN & $0,438^{* *}$ & n.s. & n.s. \\
ABA & n.s. & n.s. & n.s. \\
DMA & $-0,532^{* *}$ & n.s. & $0,331^{*}$ \\
DMC & $-0,519 * *$ & n.s. & $0,335^{*}$ \\
DED & $-0,359^{*}$ & n.s. & n.s. \\
CVD & n.s. & $0,437 * *$ & n.s. \\
DOM & $-0,417^{* *}$ & n.s. & n.s. \\
HMA & $-0,592^{* *}$ & n.s. & n.s. \\
HMC & $-0,591 * *$ & n.s. & n.s. \\
DEA & $-0,443^{* *}$ & n.s. & n.s. \\
CVA & n.s. & n.s. & n.s. \\
HDA & $-0,601 * *$ & n.s. & n.s. \\
IHA & $0,380^{*}$ & n.s. & n.s. \\
ICZ & n.s. & n.s. & n.s.
\end{tabular}

$\overline{\text { Niveaux de signification (s) : } * \mathrm{~s}>95 \% ; * * \mathrm{~s}>99 \% \text {; n.s., non significatif. }}$

d'Os Ancares (Doiras et Pedrafita) et le Quercus robur au sein de plusieurs parcelles de la province de Lugo (Tab. I et Fig. 1) dont le climat océanique hyper-humide est moins propice au Quercus pyrenaica $[2,44]$.

\subsection{Habitat climatique du Quercus pyrenaica en Galice}

L'habitat climatique central des forêts de chêne tauzin galicien (Fig. 3) se caractérise par des précipitations annuelles qui oscillent entre 820 et $1390 \mathrm{~mm}$, avec des précipitations estivales entre 55 et $160 \mathrm{~mm}$, approximativement, ce qui indique qu'elles requièrent une certain humidité environnementale, mais à moindre mesure que pour les forêts de Quercus robur $[4,16,62]$. Les valeurs de l'indice hydrique (IH) indiquent l'existence d'un climat humide [63]. Malgré ces données, dans les parcelles où l'influence méditerranéenne est plus importante, il existe une sécheresse estivale qui dure entre 1 et 3 mois [44] (Fig. 3). Quant au régime thermique, il existe une faible amplitude des valeurs des paramètres thermiques considérés, excepté la TmA $[14,50]$. Ce qui permet de considérer le climat comme étant de type mésothermique [64] (Fig. 3), selon les valeurs de l'ETP.

Les intervalles de l'habitat climatique marginal mettent en évidence l'existence de parcelles dans des zones à précipitations estivales faibles et à TMA supérieure à $25^{\circ} \mathrm{C}$, ce qui accentue davantage le caractère phyto-climatique plus méditerranéen du Quercus pyrenaica par rapport aux chênes atlantiques [2, 62].

\subsection{Habitat édaphique du Quercus pyrenaica en Galice}

Certaines variables édaphiques ne permettent pas de déterminer la limite supérieure de l'habitat central du chêne tauzin à cause de l'amplitude des intervalles marginaux supérieurs [9,
$10,21]$. C'est le cas des macro-nutriments tel que le phosphore et le calcium, que l'on considère leurs valeurs totales comme leurs valeurs superficielles. Concernant l'habitat édaphique central, les caractéristiques les plus notables sont les suivantes (Fig. 4):

- Les sols de nature siliceuse et d'acidité élevée sont prédominants, demeurant ancrés entre les classes acides très fortes et modérées [67]. Approximativement $50 \%$ des sols sont du type régosol sombrique, $24 \%$ du type brunisol humique, et $18 \%$ du type cambisol dystrique [22]. C'est-àdire que la majorité des forêts de chênes tauzins inventoriés s'installent sur des sols moins évolués que ceux des bois de Quercus robur [20, 21].

- Le pourcentage de matière organique présente une faible variation entre les valeurs totales et superficielles (Fig. 4). La composition granulométrique existante unie aux faibles valeurs de MO (de rares parcelles dépassent les $15 \%$, le long des $20 \mathrm{~cm}$ supérieurs) donnent lieu à des sols à faible capacité de solidification et très perméables [24, 45].

- Le rapport $\mathrm{C} / \mathrm{N}$ se comporte de façon similaire à la $\mathrm{MO}$ concernant la faible variation entre les valeurs totales et superficielles (Fig. 4), avec des valeurs moyennes de l'ordre de 18, dans les deux cas. Malgré ces données, les faibles valeurs du $\mathrm{pH}$ ne permettent pas de réunir les conditions optimales d'humidification, laissant apparaître un humus généralement de type moder [14].

- La concentration de phosphore présente des valeurs similaires (Fig. 4) aux donnés obtenues par d'autres auteurs en ce qui concerne ce type de formations [25, 59]. Ceci semble indiquer que les zones actuellement occupées par les forêts de chênes tauzins, n'ont pas été l'objet d'un changement quant à l'utilisation du sol, comme par exemple des cultures anciennes et de manière continue $[8,60]$. Le reste des macro-nutriments présente des valeurs supérieures à celles observées auprès des bois de Quercus robur [21], excepté pour l'azote (Fig. 4), dont les données sont semblables à celles rapportés par [24, 59].

\subsection{Aspects dendrométriques et sylvicoles des forêts galiciennes de $Q$. pyrenaica}

Le taillis est la forme principale de peuplement dominant dans ces formations $[8,11,14]$, cette méthode sylvicole présente un degré artificiel élevé, dû au fait que sa persistance dépend d'une attention soigneuse et continue [13,38]. D'autre part, l'abandon de l'exploitation traditionnelle de bois de chauffage et de charbon végétal, dû à la perte de valeur de ces produits et au dépeuplement rural, a provoqué l'arrêt des coupes à blanc de ces bois, occasionnant dans la plupart des cas, un dépassement de l'âge d'exploitation [11, 58].

L'analyse des statistiques des paramètres sylvicoles (Tab. III) démontre une haute variabilité des paramètres DEN et IHA à coefficients de variation supérieurs respectivement, à 67 et $85 \%$. La forte densité existante, de valeur moyenne supérieure à 1700 pieds/ha, dénote la présence de jeunes peuplements abandonnés, provenant d'anciennes coupes à blanc, où aucune sélection de tiges n'a été réalisée [8,29]. Les valeurs de l'indice de Hart sont en accord avec les données de densité [30, 48, 52]. Le reste des paramètres présente une variabilité moindre, spécialement ceux qui sont liés à la variable de hauteur [21]. 
La situation actuelle de ces forêts pose le problème de leur reconversion, sous le double aspect sylvicole et économique. Du point de vue sylvicole, la situation se caractérise par [3, 58] : (1) un arrêt de la croissance diamétrale et longitudinale ; (2) une épaisseur entravée, avec une présence de buissons héliophiles (Cytisus multiflorus, Erica australis, Genistela tridentata, ...) et d'arbres de petit diamètre, dont certains étant morts [4, 14] entraînent une situation de haut risque d'incendie ; (3) une rare fructification et des conditions défavorables au développement des semis ; (4) un risque de dépérissement végétatif par vieillissement des souches. Quant au point de vue économique, on essaiera de trouver des alternatives de production plus rentable que les traditionnelles : (1) une maintenance des taillis pour les forêts homogènes et de qualité, où perdure, l'exploitation de bois de chauffage ; (2) une conversion en futaie régulière ; (3) une amélioration sylvicole et pastorale, dans les zones où il existe une importante activité d'élevage ; (4) une restauration des forêts les plus dégradés en les repeuplant d'autres feuillus ou conifères.

\subsection{Relation entre les paramètres dendrométriques/ sylvicoles et le biotope}

À partir des paramètres dendrométriques/sylvicoles significatifs obtenus au cours des analyses bivariables (Tab. V), il nous a fallu éliminer le paramètre HDA et par conséquent, l'indice de Hart qui en découle [30, 52]. Cette situation est la conséquence de traitements sylvicoles inappropriés, subis par beaucoup de ces forêts, tout comme la taille en têtard des arbres, réalisée dans le but d'alimenter le bétail ou d'obtenir du bois de chauffage [3, 8]. La DED et la DEA sont des paramètres inadéquats pour nous fournir une explication concernant la situation sylvicole actuelle des forêts de Quercus pyrenaica en Galice [21, 53]. C'est pourquoi, nous ne sélectionnerons que les paramètres sylvicoles suivants : DEN, DMA, DMC et DOM, qui, dans leur totalité, sont liés au paramètre altitude (s>99\%), ainsi qu'aux précipitations estivales (s > $95 \%$ ) (Tab. VI), ce qui se confirme au cours des analyses de variances (Fig. 5).

Dans le cas de l'altitude, lorsque nous dépassons les $500 \mathrm{~m}$, le nombre de pieds est significativement plus élevé, présentant des différences supérieures à 1000 pieds/ha. De la même manière, les diamètres sont moindres, avec des différences que se situent entre 5 et plus de $10 \mathrm{~cm}$, se rapportant aux DMA, DMC ou au DOM (Fig. 5). Ce comportement s'explique par le fait qu' actuellement, beaucoup de ces forêts sont de jeunes peuplements provenant de régénération naturelle par drageonnement, à la suite d'un incendie [29, 58], alors qu'aucune sélection des tiges n'a été réalisée. En conséquence, la concurrence qui s'effectue entre les pieds provoque une stagnation et une perte de vigueur végétative [3,8]. La corrélation entre les précipitations estivales et les paramètres DMA et DMC (Fig. 5) est tout aussi significative, nous révélant que le station forestière idéale pour le Quercus pyrenaica est celle qui présente des valeurs de PE entre 125 et $150 \mathrm{~mm}[2,16]$. Les chiffres situés hors de cet intervalle supposent une diminution notable de la croissance en diamètre, qui peut être supérieure à $8 \mathrm{~cm}$; quant au DMA et au DMC, leurs valeurs peuvent s'interpréter comme le besoin modérée d'humidité estivale, étant donné le caractère plus méditerranéen de l'espèce par rapport à celui des chênes atlantiques comme le Quercus robur et le $Q$. petraea [2, 44].
En guise de conclusion, et en accord avec les résultats obtenus par d'autres auteurs, pour le Quercus pyrenaica et pour d'autres espèces $[5,10,21]$, nous avons signalé que la distribution du chêne tauzin en Galice dépend principalement des caractéristiques topo-climatiques du terrain, alors que les caractéristiques édaphiques n'entrent pratiquement pas en jeu, étant donné surtout, le fait que le substrat sur lequel il s'établisse est très semblable.

Remerciements : Ce travail a été financé par la « Dirección Xeral de Investigación e Desenvolvemento da Consellería de Innovación, Industria e Comercio da Xunta de Galicia » dans le cadre des projets de recherche PGIDT99MA29101 et PGIDIT02RFO29101PR développés par le « Departamento de Ingenería Agroforestal de la Universidad de Santiago de Compostela ».

\section{RÉFÉRENCES}

[1] Allué-Andrade J.L., Atlas Fitoclimático de España. Taxonomías, Ministerio de Agricultura, Pesca y Alimentación, INIA, Madrid, 1990.

[2] Allué M., Posición fitoclimática general de Quercus pyrenaica Willd., Studia Oecologica VIII (1991) 185-193.

[3] Allué M., Ordenación de masas de Quercus pyrenaica Willd., Cuadernos de la Sociedad Española de Ciencias Forestales 1 (1995) $107-135$.

[4] Amaral J., Quercus, in: Castroviejo S. et al. (Eds.), Flora Ibérica II, CSIC, Madrid, 1990.

[5] Aramburu M., Escribano R., Martínez E., Sáenz D., Análisis de la distribución de Quercus pyrenaica Willd en el Sistema Central, ETSIM, Madrid, 1984.

[6] Assmann E., The principles of forest yield study, Pergamon Press, Oxford, New York, 1970.

[7] Aussenac G., Interactions between forest stands and microclimate: Ecophysiological aspects and consequences for sylviculture, Ann. For. Sci. 57 (2000) 287-301.

[8] Barrio M., Díaz-Maroto I.J., Rojo A., Gestión de rebollares en montes de U.P. en el P.N. del Lago de Sanabria (Zamora), Revista Montes 62 (2000) 21-28.

[9] Blanco A., Rubio A., Sánchez O., Elena R., Gómez V., Graña D., Autoecología de los castañares de Galicia (España), Invest. Agrar. Sist. Recur. For. 9 (2000) 337-361.

[10] Bravo-Oviedo A., Montero G., Site index in relation to edaphic variables in stone pine (Pinus pinea L.) stands in Southwest Spain, Ann. For. Sci. 62 (2005) 61-72.

[11] Cañellas I., Del Río M., Roig S., Montero G., Growth response to thinning in Quercus pyrenaica Willd. coppice stands in Spanish central mountain, Ann. For. Sci. 61 (2004) 243-250.

[12] Carballeira A., Devesa C., Retuerto R., Santillan E., Ucieda F., Bioclimatología de Galicia, Xunta de Galicia-Fundación Barrie de la Maza, 1983.

[13] Carvalho J., Loireiro A., Stool and root resprouting according to different cutting seasons in a Quercus pyrenaica Willd. coppice, Ann. Ist. Sper. Selvicoltura 27 (1996) 83-88.

[14] Carvalho J. (Ed.), O Carvalho Negral, Universidade Tras-Os-Montes e Alto Douro, Vila Real, Portugal, 2005.

[15] Castroviejo M., Fitoecología de los montes de Buio y Sierra del Xistral, Lugo, Consellería de Agricultura, Gandería e Pesca, Xunta de Galicia, 1988.

[16] Ceballos L., Ruiz de la Torre J., Árboles y arbustos, ETSIM, Madrid, 1979.

[17] Claessens H., Pauwels D., Thibaut A., Rondeux J., Site index curves and autoecology os ash, sycamore and cherry in Wallonia, Southern Belgium, Forestry 72 (1999) 171-182.

[18] Daget P., Godron M., Analyse fréquentielle de l'écologie des espèces dans les communautés, Masson, 1982.

[19] DGCONA, Tercer Inventario Forestal Nacional, Galicia, A Coruña, Lugo, Ourense y Pontevedra, Ministerio de Medio Ambiente, 2001. 
[20] Díaz-Maroto I.J., Estudio ecológico y dasométrico de las masas de carballo (Quercus robur L.) en Galicia, Tesis doctoral, Univ. Politécnica de Madrid, 1997.

[21] Díaz-Maroto I.J., Vila P., Silva-Pando F.J. Autécologie des chênaies de Quercus robur L. en Galice (Espagne), Ann. For. Sci. 62 (2005) 737-749.

[22] FAO, World Reference Base for Soil Resources, World Soil Resources Reports, 84, 1998.

[23] Gaines S.D., Denny M.W., The largest, smallest, highest, lowest, longest and shortest: extremes in ecology, Ecology 74 (1993) $1677-1692$.

[24] Gallardo J.F., Santa Regina I.S., Harrison A.F., Howard D.M., Organic matter and nutrient dynamics in three ecosystems of the "Sierra de Bejar" mountains (Salamanca Province, Spain), Acta Oecol. 16 (1995) 447-459.

[25] Gallego H.A., Santa Regina I.S., Rico M., Rapp M., Variación estacional de la concentración de nutrientes en hojas y ramas en bosques naturales de Quercus pyrenaica Willd. (Sierra de Gata, España), in: Gallardo J.F. (Ed.), Proc. XI Simposium Internacional de Biogeoquímica Ambiental, 1994, pp. 111-120.

[26] Gandullo J.M., Ensayo de evaluación cuantitativa de la insolación en función de la orientación y de la pendiente del terreno, Comun. INIA Ser. Recur. Nat. 1 (1974) 95-107.

[27] Gandullo J.M., Bañares A., Blanco A., Castroviejo M., Fernández A., Muñoz L., Sánchez O., Serrada R., Estudio ecológico de la laurisilva canaria, ICONA, 1991.

[28] Gaussen H., Détermination des climats par la méthode des courbes ombrothermiques, C. R. Acad. Sci. 240 (1955) 642-644.

[29] Grandas J.A., Díaz-Maroto I.J., Silva-Pando F.J., Indicadores selvícolas de la calidad de estación de los bosques gallegos de Quercus pyrenaica Willd., Actas II Congreso Forestal Español, Mesa Temática 4 (1997) 339-344.

[30] Hart H.M.F., Stamtal en dunning; een orienteerend onderzoek naar de beste plantwijdte en duningswijze loor den djati, Veenman \& Zonen, 1928.

[31] Hill M.O., Decorana: A fortran program for detrended correspondence analysis and reciprocal averaging, Cornell Univ., New York, 1979.

[32] Hill M.O., Twinspan: A fortran program for arranging multivariate data in an ordered two-way table by classification of the individuals and attributes, Cornell Univ., New York, 1979.

[33] Hill M.O., Bunce R.G.H., Shaw M.W., Indicator species analysis: a divisive polythetic method of classification, and its application to a survey of native pinewoods in Scotland, J. Ecol. 63 (1975) 597613.

[34] Hix D.M., Multifactor classification and analysis of upland hardwood forest ecosystems of the Kichapoo River watershed, southwestern Wisconsin, Can. J. For. Res. 18 (1988) 1405-1415.

[35] Hubert A., Les types de stations forestières du Lannemezan, Gers et Moyen-Adour, Cemagref, Études Forêt 8, Bordeaux, 1991.

[36] Hummel F., Code of Sample Plot Procedure, Forestry Commission 34, 1959.

[37] ICONA, Primer Inventario Forestal de España, Ministerio de Agricultura, Madrid, 1975.

[38] Johnson P.S., Shifley S.R., Rogers R., The ecology and silviculture of oaks, CABI Publishing, 2002.

[39] Kent M., Coker P., Vegetation description and analysis. A practical approach, John Wiley \& Sons, New York, 1996.

[40] Lebourgeois F., Cousseau G., Ducos Y., Climate-tree-growth relationships of Quercus petraea Mill. stand in the forest of Bercé ("Futaie des Clos", Sarthe, France), Ann. For. Sci. 31 (2004) 361372.

[41] Le Goff N., Indice de productivité des taillis-sous-futaie de chêne dans la région Centre, Ann. Sci. For. 41 (1984) 1-34.

[42] Martínez J.E., Ayuga E., González C., Estudio comparativo de distintas funciones núcleo para la obtención del mejor ajuste según el tipo de datos, Qüesttió 16 (1992) 3-26.

[43] Mesón $M^{\mathrm{a}}$.L., Aspectos botánicos y fenológicos de Quercus pyrenaica Willd., Bol. Estac. Cent. Ecol. 22 (1982) 15-22.
[44] Mesón Ma.L., Aspectos autoecológicos de Quercus pyrenaica Willd.: distribución y climatología, Bol. Estac. Central de Ecología 23 (1983) 25-33.

[45] Mesón $M^{a}$.L., La influencia sobre el suelo de Quercus pyrenaica Willd., Bol. Estac. Central de Ecología 24 (1983) 21-26.

[46] Moreno G., Gallardo J.F., Cuadrado S., Deposición atmosférica de bioelementos y su modificación por la cubierta vegetal en bosques de Quercus pyrenaica de la Sierra de Gata (Salamanca), in: Gallardo J.F. (Ed.), Proc. XI Simposium Internacional de Biogeoquímica Ambiental, 1994, pp. 201-215.

[47] Moreno G., Gallardo J.F., Cuadrado S., Hernández J., Dinámica estacional de la humedad edáfica en bosques de Quercus pyrenaica, considerando un gradiente pluviométrico, in: Gallardo J.F. (Ed.), Proc. XI Simposium Internacional de Biogeoquímica Ambiental, 1994, pp. 163-168.

[48] Pardé J., Bouchon J., Dendrométrie, ENGREF, $2^{e}$ ed., Nancy, 1988.

[49] Pisces Conservation L.T.D., Community Analysis Package version 1.42. A program to search for structure in ecological community data, England, 1999.

[50] Retuerto R., Carballeira A., Phytoecological importance, mutual redundancy and phytological threshold values of certain climatic factors, Vegetatio 90 (1990) 47-62.

[51] Rivas-Martínez S., Memoria y mapas de series de vegetación de España, ICONA, Ministerio de Agricultura, Pesca y Alimentación, Madrid, 1987.

[52] Rondeux J., La mesure des arbres et des peuplements forestiers, Les Presses Agronomiques de Gembloux, 1993.

[53] Rubio A., Escudero A., Gandullo J.M., Sweet chestnut silviculture in a ecological extreme of its range in the west of Spain, Extremadura, Ann. Sci. For. 54 (1997) 667-680.

[54] Ruiz de la Torre J., Mapa Forestal de España, Dirección General de Conservación de la Naturaleza, Ministerio de Medio Ambiente, Madrid, 1991.

[55] Russell J.S., Moore A.W., Comparison of different depth weightings in the numerical analysis of anisotropic soil profile data, Proc. 9th. Int. Cong. Soil Sci. 4, 1968, pp. 205-213.

[56] Ryan T.P., Modern regression methods, John Wiley \& Sons, 1997.

[57] SAS Institute, SAS/STAT User's Guide, Version 8, SAS Institute Inc. Cary, North Carolina, 1999.

[58] Serrada R., González Doncel I., López Peña C., Marchal B., San Miguel A., Tolosana E., Tipificación dasométrica de los rebollares (Quercus pyrenaica Willd.) de la Comunidad de Madrid. Alternativas silvopastorales. Diseño de un plan experimental, Actas I Congreso Forestal Español, Tomo II, 1993, pp. 623-629.

[59] Santa Regina I.S., Gallardo J.F., Rico M., Martín A., Gallego H.A., Moreno G., Cuadrado S., Datos preliminares sobre biomasa aérea, producción y características edafoclimáticas de ecosistemas forestales de Quercus pyrenaica (Sierra de Gata, Salamanca), III Jornadas de la AEET, León, 1991.

[60] Silva-Pando F.J., Rigueiro A., 1992, Guía das árbores e bosques de Galicia, Galaxia, 1992.

[61] Ter Braak C.J.F., Canonical community ordination. Part I: Basic theory and linear methods, Ecoscience 1 (1994) 127-140.

[62] Timbal J., Aussenac G., An overview of ecology and silviculture of indigenous oaks in France, Ann. Sci. For. 53 (1996) 649-661.

[63] Thornthwaite C., Mather J., The water balance, Climatology 8 (1955) 1-104.

[64] Thornthwaite C., Mather J., Instructions and Tables for Computing Potential Evapotranspiration and the Water Balances, New Jersey, 1957.

[65] Valle F., Gómez-Mercado F., Mota J., Los robledales de la Sierra de Segura, Anal. Jard. Bot. Madrid XLV (1988) 247-257.

[66] Walpole R.E., Myers R.H., Myers S.L., Probabilidad y estadística para ingenieros, $6^{\mathrm{e}}$ ed., Prentice Hall, Londres, 1999.

[67] Wilde S.A., Forest soils and forest growth, Chronica Botanica Comp., 1946.

[68] Xunta de Galicia, Avance do Segundo Inventario Forestal Nacional, 1988. 